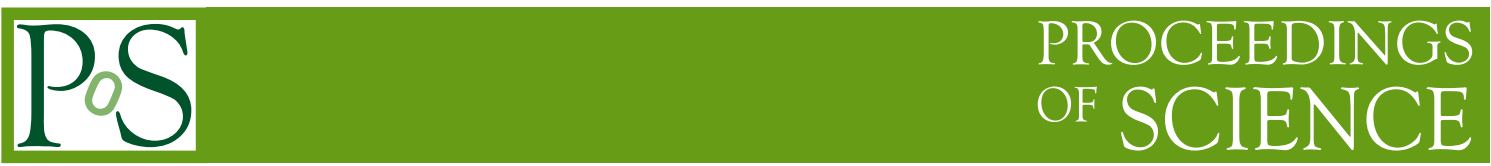

\title{
Recent results from the CERN NA60 experiment
}

\section{Pietro Cortese* for the NA60 Collaboration}

Università del Piemonte Orientale, Alessandria and INFN

E-mail: cortese@to.infn.it

\begin{abstract}
NA60 is a CERN SPS experiment that studied dimuon production in In-In collisions at $158 \mathrm{~A} \cdot \mathrm{GeV}$ per nucleon. More recently proton nucleus collisions have also been investigated in order to provide a reference for heavy ion studies. Some of the most important results obtained by the experiment are the first measurement of the $\rho$ spectral function in nuclear collision and the study of its medium-induced modifications, the production of thermal muon pairs in the invariant mass region between the $\phi$ and the $\mathrm{J} / \psi$, and the study of the $\mathrm{J} / \psi$ suppression. Recent results include further studies on the characterization of thermal pairs, the comparison of $\phi$ production in $\mu \mu$ and $K K$ channels (the $\phi$ puzzle) and the first measurement of cold nuclear matter effects on $\mathrm{J} / \psi$ production at $158 \mathrm{GeV}$, the same energy where the anomalous $\mathrm{J} / \psi$ suppression has been observed.
\end{abstract}

European Physical Society Europhysics Conference on High Energy Physics, EPS-HEP 2009,

July 16 - 222009

Krakow, Poland

${ }^{*}$ Speaker. 
NA60 is a fixed target experiment [1] which has been carried out at the CERN SPS. The main objective of the experiment is the study of strongly interacting matter in extreme conditions of temperature and pressure that, according to the predictions of Quantum Chromo Dynamics, are necessary for the transition of hadronic matter to a deconfined state of quarks and gluons. The required energy density of the order of $\sim 1 \mathrm{GeV} / \mathrm{fm}^{3}$ can be reached in the laboratory only in the environment produced in relativistic heavy-ion collisions.

NA60 is composed of three main detectors. The Muon Spectrometer (MS), inherited from NA50, is made of a hadron absorber, an air core toroidal magnet, 8 MWPC muon tracking stations and a system of scintillator hodoscopes for muon triggering. It is complemented by a Zero Degree Calorimeter and by a radiation-hard silicon pixel Vertex Telescope that is the most innovative element in the experiment. It is placed immediately downstream the target, into a $2.5 \mathrm{~T}$ dipole field in the acceptance of the MS and allows a precise measurement of the track momenta before they undergo multiple scattering in the hadron absorber. Combining the information of the tracker with the one from the MS there is a strong improvement in the measurement of the muon kinematics. This brings an improvement of the mass resolution, the possibility to identify muons from offtarget decays and a considerable rejection of the combinatorial background. The selective trigger and high luminosity allowed the experiment to collect a high statistics dimuon sample.

The experimental program involved the study of In-In collisions, a complementary system with respect to previous studies by NA50, and also p-A collisions that are used as a reference for heavy-ion studies. In particular NA60 measured p-A collisions at 400 and $158 \mathrm{GeV}$ incident energy, allowing for the first time the comparison of A-A and p-A results taken at the same energy.

\section{Low and intermediate masses}

Previous results by NA60 include the precise determination of the dimuon excess present in the region $m_{\mu \mu}<m_{\phi}$. The high statistics and data quality allowed not only to confirm the observation of the excess made by CERES experiment [2], but to characterize the nature of the excess [3]. NA60 extracted its absolute yield [4] and its dependence on the different kinematical variables: mass, rapidity, $p_{T}[5]$ and, more recently, polarization [6].

Concerning the study of the "intermediate mass region", i.e. the region between the $\phi$ and the $\mathrm{J} / \psi$, the off-vertex decays tagging capabilities of NA60 allowed to establish that the excess already observed by NA50 [7] is of prompt origin [8] and no charm enhancement is required to describe the data.

The analysis of $p_{T}$ spectra [5] provides additional information on the origin of the observed dilepton excess because the dynamics of the reaction is encoded in this variable. For masses lower than $1 \mathrm{GeV}$ the effective temperature rises linearly with mass (see Figure 1) and this is reminiscent of the flow of a hadronic source. The lower mass resonances are aligned along the temperature trend established by the continuum, except for the $\rho$ meson. The unmodified $\rho$ appears to collect higher flow and it is likely to be produced in the later stages of the collision history.

For masses above $1 \mathrm{GeV}$ there is a sudden drop of the effective temperature. This is naturally explained as the transition to a source of partonic origin, that contributes to the emission in the earliest stages of the collisions before the flow builds up. Excess data have been corrected for acceptance and normalized in absolute scale, allowing a direct comparison in shape and in yield with theoretical models [4]. 


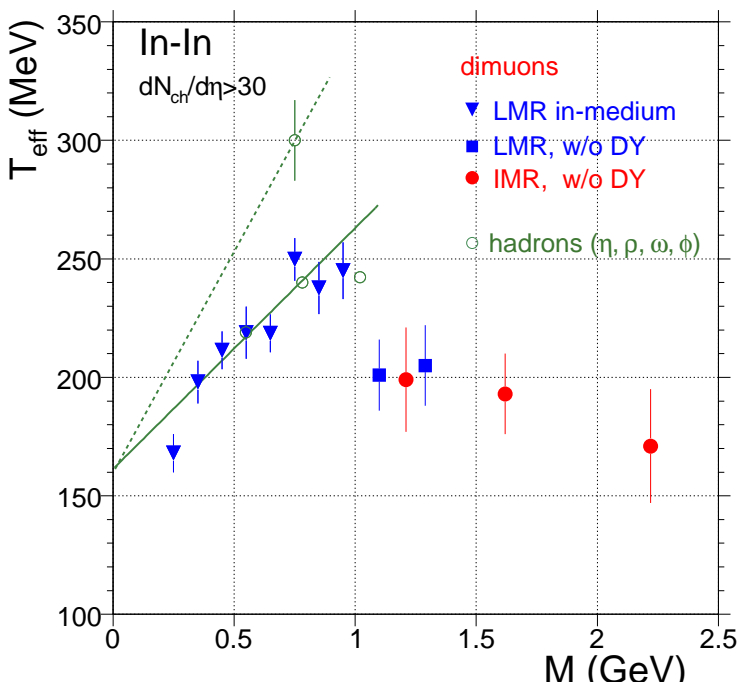

Figure 1: Inverse slope parameter $T_{\text {eff }}$ vs. dimuon mass for excess in comparison to hadrons. The "peak" $\rho$ contribution is subtracted (in-medium). DY is subtracted for $m>1 \mathrm{GeV} . D \bar{D}$ is subtracted throughout. Continuum line indicates $T_{\text {eff }}$ rise due to radial flow. Dashed line points to the freeze-out $\rho$ to guide the eye.

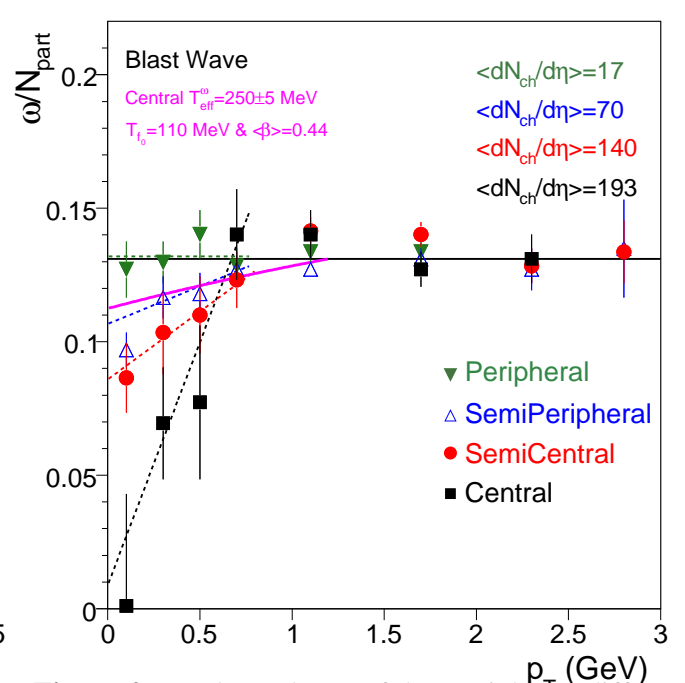

Figure 2: $p_{T}$ dependence of the $\omega$ yield for different centralities. See [4] for details.

The absence of polarization is a necessary condition for dimuon emission from a thermalized medium. The polarization analysis has been performed in the Collins-Soper and GottfriedJackson reference frames, in mass bins for the excess dimuon in the range $0.4-0.9 \mathrm{GeV} / \mathrm{c}^{2}$. Three analysis methods, having different sensitivity to systematic and statistical uncertainties, have been employed for the analysis. In the first method a 2D (two-dimensional) acceptance correction has been performed using a $[\cos \theta, \phi]$ matrix and the polarization parameters have been simultaneously estimated by fitting the data with

$$
\frac{1}{\sigma} \frac{d \sigma}{d \cos \theta d \phi}=1+\lambda \cos ^{2} \theta+\mu \sin 2 \theta \cos \phi+\frac{v}{2} \sin ^{2} \theta \cos 2 \phi
$$

All parameters were found to be consistent with 0 . The second approach involves the same 2D acceptance correction but 1D fits on the spectra projected on the two axes and the third method is based on 1D corrections. All methods confirmed the absence of polarization supporting therefore the thermal origin of the dimuon excess as emerged from the study of $p_{T}$ and mass spectra [6].

A note-worth recent development is the analysis of the in-medium modification of the $\omega$ meson (see Figure 2). In this case the effect can't be observed as the appearance of a modified $\omega$ in some region of the invariant mass spectrum as it is masked by the much stronger dimuon yield from modified $\rho$. On the contrary it can be observed as the disappearance of the $\omega$ yield vs. centrality which exhibits a strong $p_{T}$ dependence [4]. While the $p_{T}$ spectra of the $\phi$ and of the $\omega$ at $p_{T}>0.8 \mathrm{GeV} / \mathrm{c}$ are perfectly thermal and agree very well with the "blast wave" fits at all centralities, the low- $p_{T} \omega$ spectra become gradually depleted as centrality increases, with almost complete disappearance of the $\omega$ with $p_{T}<0.2 \mathrm{GeV} / \mathrm{c}$ for the most central collisions.

One last measurement that has been possible thanks to the high statistics and resolution in this mass region is the study of the electromagnetic transition form-factors in $\eta \rightarrow \mu^{+} \mu^{-} \gamma$ and $\omega \rightarrow \mu^{+} \mu^{-} \pi^{0}$ decays in peripheral In-In collisions [9]. The results confirm (now at $10 \sigma$ level) 


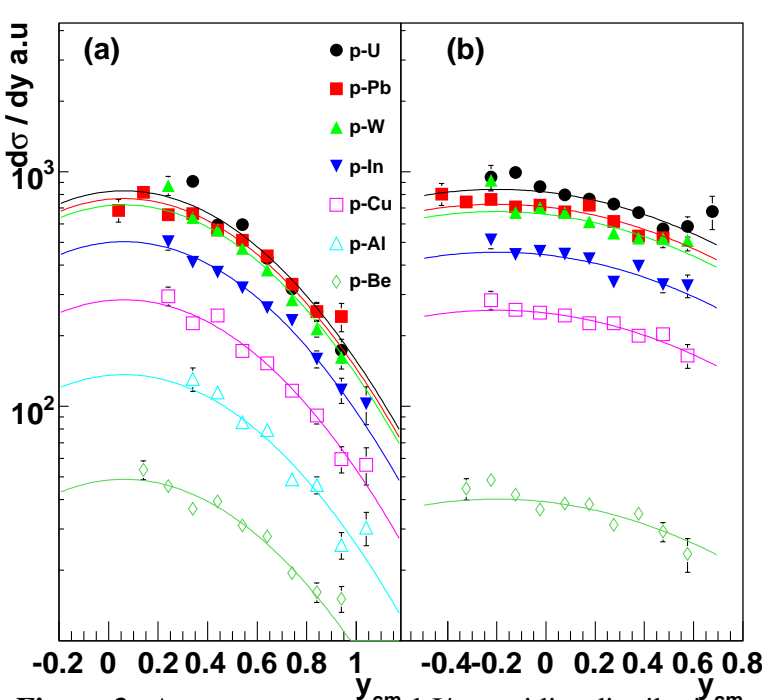

Figure 3: Acceptance corrected $\mathrm{J} / \psi$ rapidity distributiof at 158 (a) and 400 (b) $\mathrm{GeV}$ incident energy for the different targets.

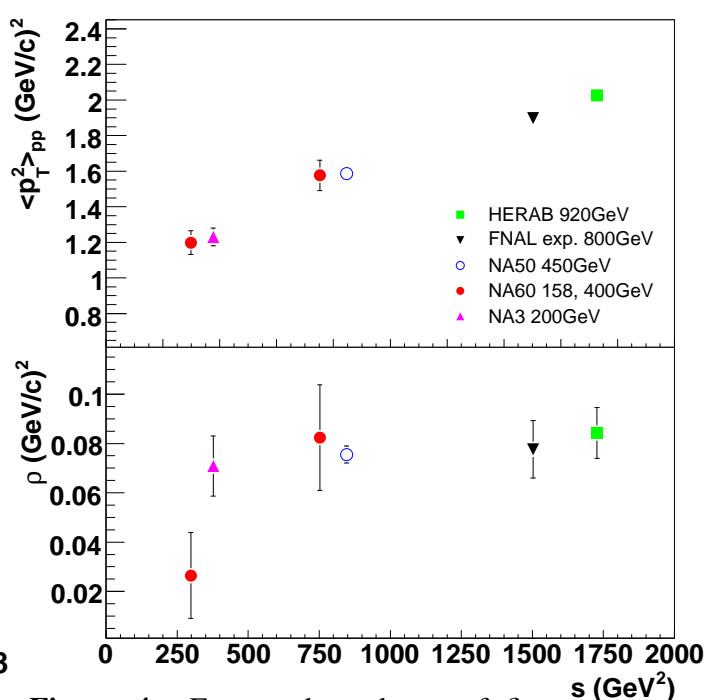

Figure 4: Energy dependence of fit parameters $\left\langle p_{T}^{2}\right\rangle_{p p}$ (top) and $\rho$ (bottom).

a strong anomaly with respect to Vector Meson Dominance calculations for the $\omega$ decay, first observed by the Lepton-G experiment [10]. The ongoing analysis of the p-A data sample will hopefully give further improvement to this measurement.

\section{$\phi$ production in $\mu^{+} \mu^{-}$and $K^{+} K^{-}$channels}

The NA60 experiment addressed, in the In-In system, the reported discrepancy between $\phi$ effective temperatures and yields measured in $\mu^{+} \mu^{-}$and $K^{+} K^{-}$channels. Thanks to the information provided by the Vertex Telescope it has been possible to study simultaneously $\phi$ production in the two channels in the same experiment. Since no PID was available, the $\phi \rightarrow K K$ analysis was based on the combination of track pairs detected in minimum bias events (with suitable kinematical cuts) assuming they are kaons. The subtraction of the huge combinatorial background from the resulting invariant mass spectrum has been performed with an event mixing technique. The NA60 results found no difference in temperature and yields between the two channels and therefore there is no " $\phi$ puzzle" in In-In collisions. A complete coverage of the results and the discussion on the comparison with NA50 and NA49 Pb-Pb results is given in $[11,12]$.

\section{$\mathrm{J} / \psi$ suppression}

The production and suppression of $\mathrm{J} / \psi$ in heavy-ion collisions is regarded as one of the key measurements to study the transition from confined matter to a plasma of quarks and gluons (QGP). It has been predicted [13] that the inter-quark potential between the $c$ and $\bar{c}$ that form the $\mathrm{J} / \psi$ meson would be screened in presence of a deconfined medium. This would result in the lowering of the $c \bar{c}$ binding energy and eventually to the dissolution of $c \bar{c}$ bound states. This idea was confirmed later by lattice simulations and potential models.

From an experimental point of view, when performing an inclusive measurement of $J / \psi$ production, the effect of deconfinement would result in a strong deviation of $\mathrm{J} / \psi$ yield from binary collision scaling. Such an effect ("anomalous J/ $\psi$ suppression") has been observed at SPS in $\mathrm{Pb}-\mathrm{Pb}$ collisions at $158 \mathrm{~A} \cdot \mathrm{GeV}$ incident energy [14], at $\mathrm{RHIC}$ in $\mathrm{Au}-\mathrm{Au}$ and $\mathrm{Cu}-\mathrm{Cu}$ collisions at $\sqrt{s}=200 \mathrm{~A} \cdot \mathrm{GeV}$ and, to a minor extent, in In-In collisions at $158 \mathrm{GeV}$ [15]. However, part of the 


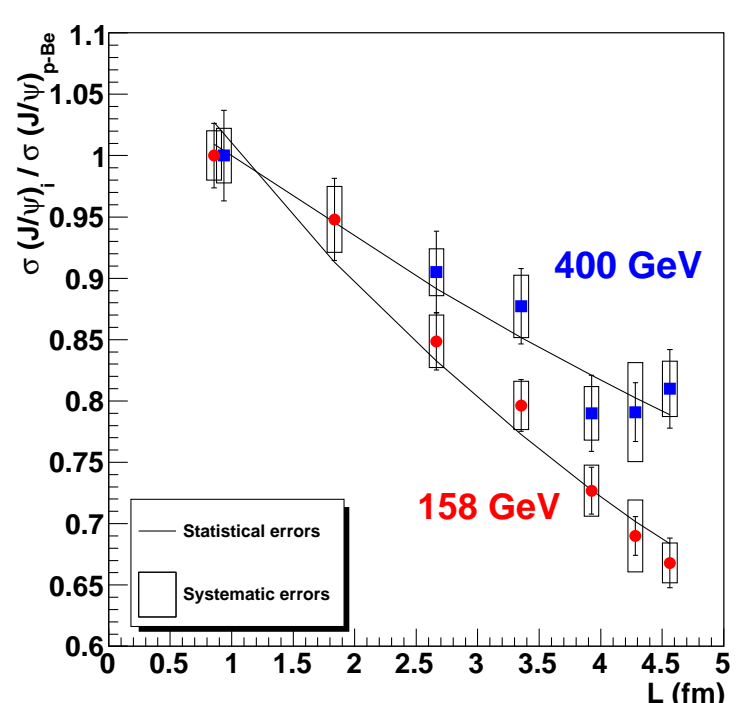

Figure 5: $\mathrm{J} / \psi$ cross section ratios for $\mathrm{p}-\mathrm{A}$ collisions at $158 \mathrm{GeV}$ and $400 \mathrm{GeV}$ as a function of $\mathrm{L}$, the average $\mathrm{J} / \psi$ path in nuclear matter.

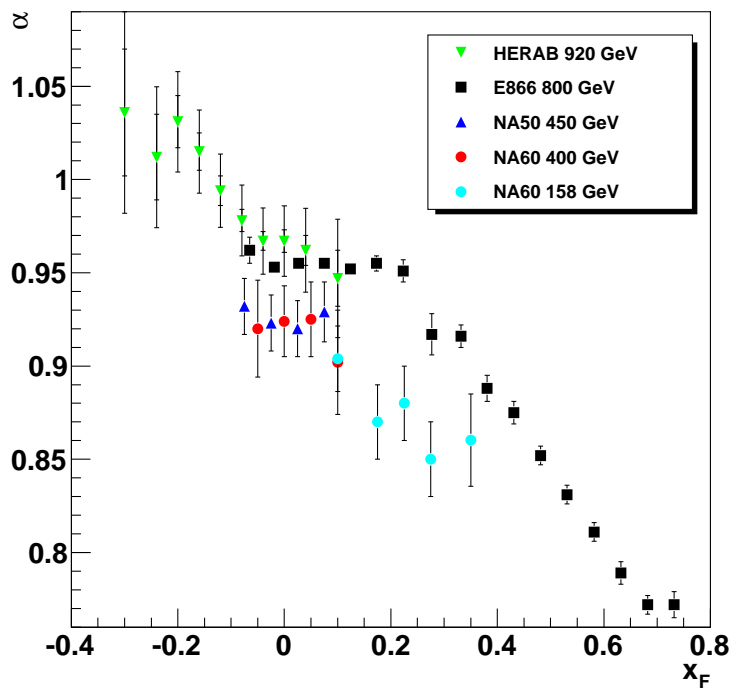

Figure 6: $\alpha$ for the $\mathrm{J} / \psi$ as a function of $x_{F}$. The energy dependence of $\alpha$ already observed by NA50 (vs. E866) is confirmed. $158 \mathrm{GeV}$ data exibits an even stronger suppression.

deviation from binary scaling has to be attributed to cold nuclear matter effects. Therefore, the identification of the suppression mechanism relies on the careful evaluation of cold-nuclear-matter effects ("normal J/ $\psi$ suppression"). These include initial state effects (shadowing) and final-state effects like the dissociation of the $\mathrm{J} / \psi$ (pre-)resonant state in the collisions with nuclear matter. These are usually parametrized, in the framework of the Glauber model, with the quantity $\sigma_{a b s}$, that summarizes all nuclear effects experienced by the $\mathrm{J} / \psi . \sigma_{a b s}$ therefore is an effective parameter as it is averaged over the different charmonia states that feed the observed $\mathrm{J} / \psi$ yield, over their time-evolution and include also initial state effects .

To evaluate cold nuclear matter effects, the NA38 and NA50 experiments have performed, in the past years, various measurements of $\mathrm{J} / \psi$ production in proton-nucleus collisions at 400 $450 \mathrm{GeV}$ incident energies [16-18]. Therefore the comparison of $\mathrm{p}-\mathrm{A}$ measurements with $\mathrm{Pb}-\mathrm{Pb}$ and $\mathrm{In}-\mathrm{In}$ results taken at $158 \mathrm{GeV}$ required the assumption that $\sigma_{a b s}$ is energy independent. A measurement of $\mathrm{J} / \psi$ production in $\mathrm{p}-\mathrm{A}$ at $158 \mathrm{GeV}$ allows a more direct comparison.

To this end the NA60 experiment has measured $\mathrm{J} / \psi$ production in p-A collisions at $158 \mathrm{GeV}$ incident energy with a setup similar to the one used for In-In studies. The most relevant modification was the use of different nuclear targets $(\mathrm{Be}, \mathrm{Al}, \mathrm{Cu}, \mathrm{In}, \mathrm{W}, \mathrm{Pb}$ and $\mathrm{U})$ that were exposed simultaneously to the proton beam. The excellent vertexing capability of the NA60 pixel telescope allows to unambiguously identify the target where the $\mathrm{J} / \psi$ has been produced by using the information of the two muon tracks only .

In Figure 3 the rapidity dependence of $\mathrm{J} / \psi$ production is shown for 158 and $400 \mathrm{GeV}$ data sets. The narrow rapidity coverage of the spectrometer and the low statistics do not allow to investigate the evolution of the rapidity shape with $A$. They are fitted with gaussian curves with common mean and width. Concerning $d \sigma / d p_{T}$ we observe a broadening of the $p_{T}$ distributions with $A$. By fitting the results with $\left\langle p_{T}^{2}\right\rangle=\left\langle p_{T}^{2}\right\rangle_{p p}+\rho\left(A^{1 / 3}-1\right)$ (that is approximately proportional to the average path of the incoming parton in nuclear matter and to the path of the $\mathrm{J} / \psi$ in its way-out from the collision region as well) we can compare the normalization and the slope with the available systematics as shown in Figure 4. The $\left\langle p_{T}^{2}\right\rangle_{p p}$ is compatible with a linear growth with $s$. For what 
concerns the slope parameter $\rho$ it seems compatible with an increase at low energies followed by a plateau.

The evaluation of cold nuclear matter effects has been performed integrating the yields over $p_{T}$, in the rapidity region $3.2<y_{l a b}<3.7$ where all the targets have good coverage. Nuclear effects are evaluated by studying the $\mathrm{J} / \psi$ cross section ratios $\sigma_{p A} / \sigma_{p B e}$, i.e. between the production on a given target of mass number $A$ w.r.t. the Be target that is the lightest one present in the setup. Since all the targets are simultaneously exposed, the beam luminosity factors cancel in the ratio (apart for a small beam attenuation that is however taken into account in the calculations), and also the trigger efficiency and the reconstruction efficiency in the Muon Spectrometer cancel. On the other end, the reconstruction efficiencies in the Vertex Telescope do not cancel completely because each target is seen under a slightly different angle. Therefore an accurate evaluation of this quantity and of its time evolution is required. In Figure 5 we show the cross section ratios for $J / \psi$ at 158 and 400 $\mathrm{GeV}$. By fitting the points within the Glauber model we obtain the following preliminary result for the $\mathrm{J} / \psi$ absorption cross section in nuclear matter: $\sigma_{a b s}(158)=7.6 \pm 0.7 \pm 0.6 \mathrm{mb}$. This value is significantly higher with respect to NA50 measurements at higher energies [16-18]. Concerning $400 \mathrm{GeV}$ data we obtain $\sigma_{a b s}(400)=4.3 \pm 0.8 \pm 0.6 \mathrm{mb}$, in good agreement with the previous NA50 measurement $4.6 \pm 0.6 \mathrm{mb}$. In Figure 6 we compare the NA60 results with previous measurements at different energies as a function of $x_{F}$. For compatibility with the other experiments the results were fitted with the usual parametrization $\sigma_{p A}=\sigma_{0} \cdot A^{\alpha}$.

The impact of the higher absorption cross section on the absorption reference for $\mathrm{Pb}-\mathrm{Pb}$ and In-In collisions has been investigated and a preliminary evaluation is given in [21].

\section{References}

[1] G. Usai et al. (NA60 Collaboration), Eur. Phys. J. C 43 (2005) 415.

[2] G. Agakichievet al.(CERES Collaboration), Eur. Phys. J. C 41, 475 (2005).

[3] R. Arnaldi et al. (NA60 Collaboration), Phys. Rev. Lett. 96 (2006) 162302.

[4] R. Arnaldi et al. (NA60 Collaboration), Eur. Phys. J. C 61 (2009) 711.

[5] R. Arnaldi et al. (NA60 Collaboration), Phys. Rev. Lett. 100 (2008) 022302.

[6] R. Arnaldi et al. (NA60 Collaboration), Phys. Rev. Lett. 102 (2009) 222301.

[7] M.C. Abreu et al. (NA38 and NA50 Collaborations), Eur. Phys. J. C 14, 443 (2000).

[8] R. Arnaldi et al. (NA60 Collaboration), Eur. Phys. J. C 59 (2009) 607.

[9] R. Arnaldi et al. (NA60 Collaboration), Phys. Lett. B 677 (2009) 260.

[10] R.I. Djeliadin et al., Phys. Lett. B 94 (1980) 548 and Phys. Lett. B 102 (1981) 296.

[11] R. Arnaldi et al. (NA60 Collaboration), accepted by Eur. Phys. J. C, hep-ex/0906.1102.

[12] A. De Falco et al. (NA60 Collaboration), proceedings of QM 2009, nucl-ex/0907.4702.

[13] T. Matsui and H. Satz, Phys. Lett. B 178 (1986) 416.

[14] B. Alessandro et al. (NA50 Collaboration), Eur. Phys. J. C 39 (2005) 335.

[15] R. Arnaldi et al. (NA60 Collaboration), Phys. Rev. Lett. 99 (2007) 132302.

[16] B. Alessandro et al. (NA50 Collaboration), Phys. Lett. B 553, (2003) 167.

[17] B. Alessandro et al. (NA50 Collaboration), Eur. Phys. J. C 33, (2004) 31.

[18] B. Alessandro et al. (NA50 Collaboration), Eur. Phys. J. C 48, (2006) 329.

[19] J. Badier et al. (NA3 Collaboration), Z. Phys C 20 (1983) 101.

[20] R. Arnaldi et al. (NA60 Collaboration), proceedings of QM 2009, nucl-ex/0907.5004.

[21] E. Scomparin et al. (NA60 Collaboration), proceedings of QM 2009, nucl-ex/0907.3682. 